\title{
Efficient 3D Object Recognition via Geometric Information Preservation
}

\author{
Hongsen Liu ${ }^{\mathrm{a}, \mathrm{b}, \mathrm{c}}$, Yang Cong ${ }^{\mathrm{a}, \mathrm{b}, *}$, Chenguang Yang ${ }^{\mathrm{d}}$, Yandong Tang ${ }^{\mathrm{a}, \mathrm{b}}$ \\ ${ }^{a}$ State Key Laboratory of Robotics, Shenyang Institute of Automation, Chinese Academy of Sciences, Shenyang, 110016, China \\ ${ }^{b}$ Institutes for Robotics and Intelligent Manufacturing, Chinese Academy of Sciences, \\ Shenyang, 110016, China \\ ${ }^{c}$ University of Chinese Academy of Sciences, 100049, China \\ ${ }^{d}$ Bristol Robotics Laboratory, University of the West of England, Bristol, BS16 1QY, UK
}

\begin{abstract}
Accurate 3D object recognition and 6-DOF pose estimation have been pervasively applied to a variety of applications, such as unmanned warehouse, cooperative robots, and manufacturing industry. How to extract a robust and representative feature from the point clouds is an inevitable and important issue. In this paper, an unsupervised feature learning network is introduced to extract 3D keypoint features from point clouds directly, rather than transforming point clouds to voxel grids or projected RGB images, which saves computational time while preserving the object geometric information as well. Specifically, the proposed network features in a stacked point feature encoder, which can stack the local discriminative features within its neighborhoods to the original point-wise feature counterparts. The main framework consists of both offline training phase and online testing phase. In the offline training phase, the stacked point feature encoder is trained first and then generate feature database of all keypoints, which are sampled from synthetic point clouds of multiple model views. In the online testing phase, each feature extracted from the unknown testing scene is matched among the database by using the K-D tree voting strategy. Afterwards, the matching results are achieved by using the hypothesis \& verification strategy. The proposed method is extensively evaluated on four public datasets and the results show that ours deliver comparable or even superior performances than the state-of-the-arts in terms of F1-score, Average of the 3D distance (ADD) and Recognition rate.
\end{abstract}

Keywords: stacked 3D feature encoder, 3D object recognition, 6-DOF pose estimation, geometric information preservation.

\section{Introduction}

3D object recognition and 6-DOF pose estimation are of great significance to many practical applications, e.g., unmanned warehouse, cooperative robots, and manufacturing in-

5 dustry [1, 2, 3, 4, 5, 6]. However, this is still a challenge due to the diverse attributes of objects, which results in the limited discrimination of handcrafted feature descriptors. In this paper, we focus on 3D object recognition and 6-DOF pose estimation of objects with texture-less or surface-smooth. The

10 lack of interesting points make them intractable to extract robust descriptors. Most classical 2D [7, 8, 9, 10, 11, 12] and 3D $[13,14,15,16,17,18,19,20]$ local feature-based methods cannot perform well on such objects for the sake of weak keypoint descriptors. Template feature-based methods [21, 22, 23, 24]

15 can achieve better recognition results for texture-less objects by extracting global features, but their performances could be deteriorated under heavy occlusion as well. While most existing patch feature-based methods [25, 26, 27] could extract

\footnotetext{
This work is supported by Nature Science Foundation of China under Grant (61722311, U1613214, 61821005, 61533015) and CAS-Youth Innovation Pro- 40 motion Association Scholarship (2012163).

* Corresponding author

Email addresses: liuhongsenesia. cn (Hongsen Liu), congyang81@gmail.com (Yang Cong), cyangeieee.org (Chenguang Yang), ytang@sia.cn (Yandong Tang)
}

various features from RGB-D images to solve such problem$\mathrm{s}$, they inevitably involve mapping the $3 \mathrm{D}$ real world into $2 \mathrm{D}$ image space and induce the loss of 3D spatial information accordingly. In comparison, [28] tries to handle this problem by converting the point clouds into regular voxel grids and extracting 3D patch features accordingly. However, this introduces unnecessary computational cost. Recently, a variety of CNNbased methods [29, 30, 31] have tried to learn features from large amounts of identically distributed training data, which depends heavily on large volume of data. The foregoing issues motivate us to extract a more efficient 3D features representation from raw point clouds directly and estimate the 6-DOF pose of the $3 \mathrm{D}$ objects depending on the hypotheses generation and verification strategy as shown in Fig. 1.

Generally, there are three main stages involved for 3D object recognition: 1) 3D feature extraction: Unlike the existing methods [25, 26, 27, 28] and motivated by [32][33], we design a 3D feature encoder, which enables point interaction within a local neighborhood sphere, by stacking the locally spherewise aggregated feature on point-wise features. Stacking multiple encoder layers allows further learning deep sphere-wise features and point-wise features. Afterwards, the point-wise features are sequentially sent to the structurally similar decoder to reconstruct the input points. The output sphere-wise features of the middle layer are used for characterizing 3D keypoints descriptor. The mean squared error (MSE) is adopted as the 


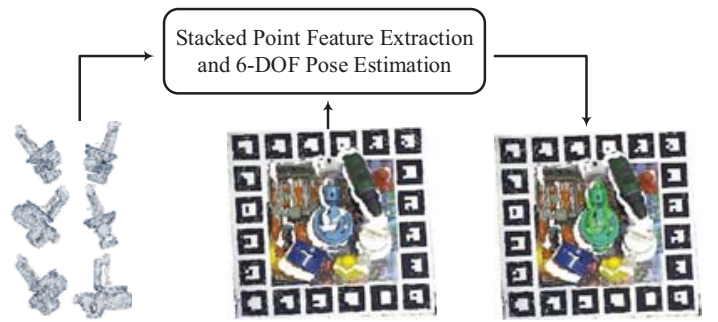

$\begin{array}{lll}\text { (A) The raw point clouds } & \text { (B) The raw point clouds } & \text { (C) The result of 6-DOF pose }\end{array}$ of model samples of real scenario

back project to $3 \mathrm{D}$ space $[7,8,9,10,11]$. These methods perform well on 3D objects with rich texture surface. However, many objects in our daily life are texture-less, especially in the industrial environment. Recently, the RGB-D sensors with 85 low cost and acceptable accuracy, e.g., Kinect, become popular. Several point clouds based features are designed depending on various local 3D surface $[13,14,15,16,17,18,19]$ without projecting feature points from $2 \mathrm{D}$ image to $3 \mathrm{D}$ space. The basic assumption of these methods is that the surface normal of o the corresponding objects have rich variations. These methods may cause ambiguity for planar or self-symmetric objects due to various repeating local surfaces [20].

Template feature-based methods: Template features are achieved from the scanning model under multi-view, and the 5 optimal matching is searched by sliding windows, which are commonly robust to texture-less objects. Line2D [22] merely employs the image contours to denote a $3 \mathrm{D}$ object with a limited set of templates and achieves efficient matching by linearizing the memory for parallelization. As an improvemen$\mathrm{t}$, LineMod [21] performs robust 3D object detection by embedding quantized image contours and normal orientations on RGB-D images. However, these methods are being scaled linearly with the number of templates. To this end, R. Rioscabrera et al. [23] optimizes the matching via a cascaded classification scheme and gets 10 times speedup. W. Kehl et al. [24] proposes an improvement approach based on LineMod template features via hashing matching.

Patch feature-based methods: Recently, some feature representation methods based on local RGB-D patches are proposed, e.g., A. Tejani et al.[25] employ a manually designed feature inspired by [21] along with random forests based voting schemes for the estimation of the 6-DOF pose. A Doumanoglou et al. [26] learn patch features via an unsupervised deep Sparse Autoencoder instead of manually designed. Given that the training classifier requires to learn the background as a negative class, the method is normally constrained as dataset-specific. Instead, W. Kehl et al. [27] train a Convolution Autoencoder to extract patch features and estimate 6-DOF pose based on K-nn search, which gives better performance. Liu et al. [28] present a 3D Voxel Autoencoder by converting the point clouds into voxel grids for fully using the 3D spatial structure information.

Trainable CNN-based methods: Even various deep learning based methods have justified their performance on object detection, classification and segmentation [32, 35, 36], these methods remain unable to accurately yield the 6-DOF object pose as a regression problem [37][38]. For 3D object recognition, the frequently-used strategy is first to segment and detect object on the RGB-D images and then back project them to 3D 30 space to acquire rough location. Eventually, the Iterative closet point (ICP) is employed to refine the 6-DOF pose based on the approximate models [39, 40, 41]. Recently, some end-to-end methods are proposed $[29,30,31]$ to predict the $2 \mathrm{D}$ bounding box in the image and compute the $6 \mathrm{D}$ pose using a $\mathrm{PnP}$ algorithm [42]. These methods inevitably rely on large amounts of identically distributed training data, which acquire extra cost in collecting these training datasets. 


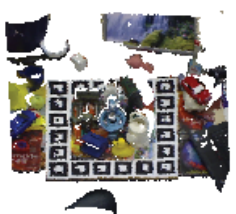

(A) Raw Point Clouds

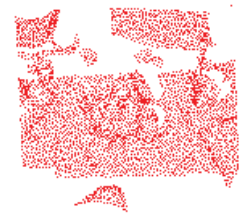

(B) Farthest Point Sampling

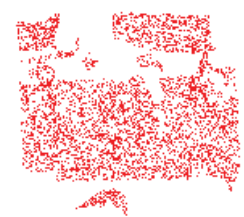

(C) Random Sampling
Figure 3: The effect of different sampling methods. (A) The raw input point clouds. (B) The effect of the Farthest Point Sampling method (FPS). (C) The effect of the Random Sampling method (RS).

\section{Methodology}

In this section, the framework of our 3D object recognition 6-DOF pose estimation method is introduced. The problem can be summarized as given a 3D model $\mathcal{M}$ of a specific object and a testing scene $\mathcal{S}$, which needs to estimate the 6-DOF pose of all the $\mathcal{M}$ existing in $\mathcal{S}$ at one time. Fig. 2 demonstrates the framework of our method. Generally, it consists of ing Phase. 1) In the offline training phase, the stacked feature encoder is trained first and then generate feature database of al1 keypoints, which are sampled from synthetic point clouds of multiple model views. Each feature holds a 6D pose annotascene are matched among the database by using the K-D tree searching. The matching results cast a collection of hypotheses that are refined via a verification strategy.

\subsection{Sphere-wise data sampling and grouping}

Typically, the point clouds of a low-cost depth sensor is composed of more than $30 \mathrm{k}$ points. Due to the density of the point clouds varies significantly in the whole space, operating directly on all points not only increases the memory/efficiency burden, but also disturb the detection accuracy. To this end, the ple keypoints as shown in Fig. 3, where FPS covers the entire surface shape better comparing with Random Sampling (RS) [44]. The sampling performance is compared in the experimental part. For a given point clouds and a support radius $r$, we first group the sphere-wise local point sets $l_{p}=\left[p_{1}, \ldots, p_{n}\right]$ with $n$ points of $r$-nearest neighboring search radius around each keypoint. The model views and scenes use different sampling number of $t$. Specifically, for spheres with a point number more than ple $n$ points respectively. In our case, the keypoints sampling number $t_{m}=512$ for model views and $t_{s}=4096$ for testing scenes. The local points sampling number $n=256$ with a support radius $r$, which is set as $\frac{1}{3}$ of the shortest edge of the $3 \mathrm{D}^{215}$

\subsection{Stacked Point Feature Encoder}

In this subsection, the process will be elaborated, which ex-220 tracts the sphere-wise feature descriptor via the encoder layers. The offline training phase of Fig. 2 illustrates the hierarchical

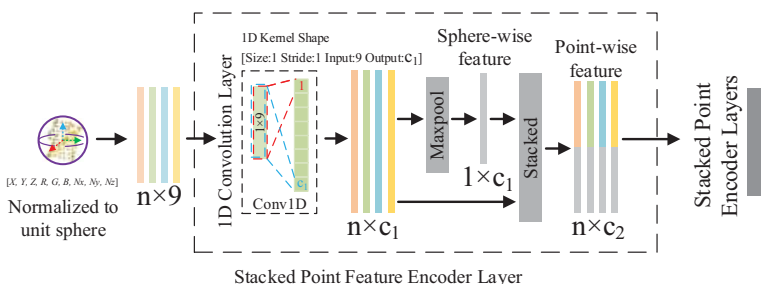

Figure 4: The architecture of the Stacked Point Feature Encoder Layer, which consists of a 1D Convolution Layer for feature transformation, a Maxpool Layer for feature aggregation and a Stacked Feature Layer for feature concatenation. The following stacked encoder layers yield deep features layer by layer.

feature encoding process. The architecture details are described in the following paragraph as shown in Fig. 4.

Denote $S=\left\{l_{p_{i}}=\left[X_{i}, Y_{i}, Z_{i}, R_{i}, G_{i}, B_{i}, N_{i}\right]^{T} \in\right.$ $\left.\mathbb{R}^{9}\right\}_{i=1 \ldots n}$ as a local sphere containing $n$ points, where $l_{p_{i}}$ contains $X Y Z$ coordinates and $R G B$ values for the $i$-th point and $N_{i}$ is the calculated normal direction, which consists of $N_{x}, N_{y}, N_{z}$. It needs to be explained that the normals are computed before the sampling and grouping of the spheres so as to ensure the continuity of the surface shape of the input point clouds. Firstly, each sphere is normalized, including centralization and normalization of coordinates, normalization of colors and unitization of normal vectors. Next, the normalized spheres with the size as $[b \times n \times 9]$ are transformed through the fully connected layers $(\mathrm{FC})$ into aggregated feature space with the size as $\left[b \times n \times c_{1}\right]$, where $b$ represents the training batch size 95 (in our case, $b=4$ ), $n$ represents the number of points, 9 is the feature dimension of the initial point-wise features, $c_{1}$ is the dimension of transformed features. Specifically, in order to process 3D point clouds more efficiently, 1D convolution with [1] kernel size and [1] kernel stride are used to replace the fully connected layer to transform the input data into $\left[b \times n \times c_{1}\right]$. To extract sphere-wise features, which represent the global features of the local 3D points sphere, the max pooling layer is used as a symmetric function that aggregates information from all the point-wise features to achieve sphere-wise features with the size as $\left[b \times 1 \times c_{1}\right]$. The use of max pooling layer is not only to aggregate the spatial dimension of features, but also to ensure the permutations invariance of the unordered 3D points. Afterwards, the sphere-wise features are fed back to per point-wise features by stacking the sphere-wise features on each of the point-wise features with size as $\left[b \times n \times c_{2}\right]$, where $c_{2}=2 \times c_{1}$. Through this way, the new stacked point-wise features are able to preserve both the local and global information.

We use $\left[c_{i n}^{i}, c_{\text {out }}^{i}\right]$ to represent the I/O of $i$-th encoder layer that transforms input features of dimension $c_{i n}$ into output features of dimension $c_{\text {out }}$. For the first encoder layer, $c_{i n}^{1}$ is 9 that represents there are 9 dimensions of the raw points clouds attributes. For each encoder layer, there are two output features, i.e., the sphere-wise feature $c_{\text {out }}^{\text {sphere }}$ and the new stacked point-wise feature $c_{\text {out }}^{\text {stacked }}$. Only $c_{\text {out }}^{\text {stacked }}$ could be transformed through next encoder layer into deeper sphere-wise and pointwise features; the $c_{\text {out }}^{\text {sphere }}$ could only be extracted from the end of the encoder layers. 


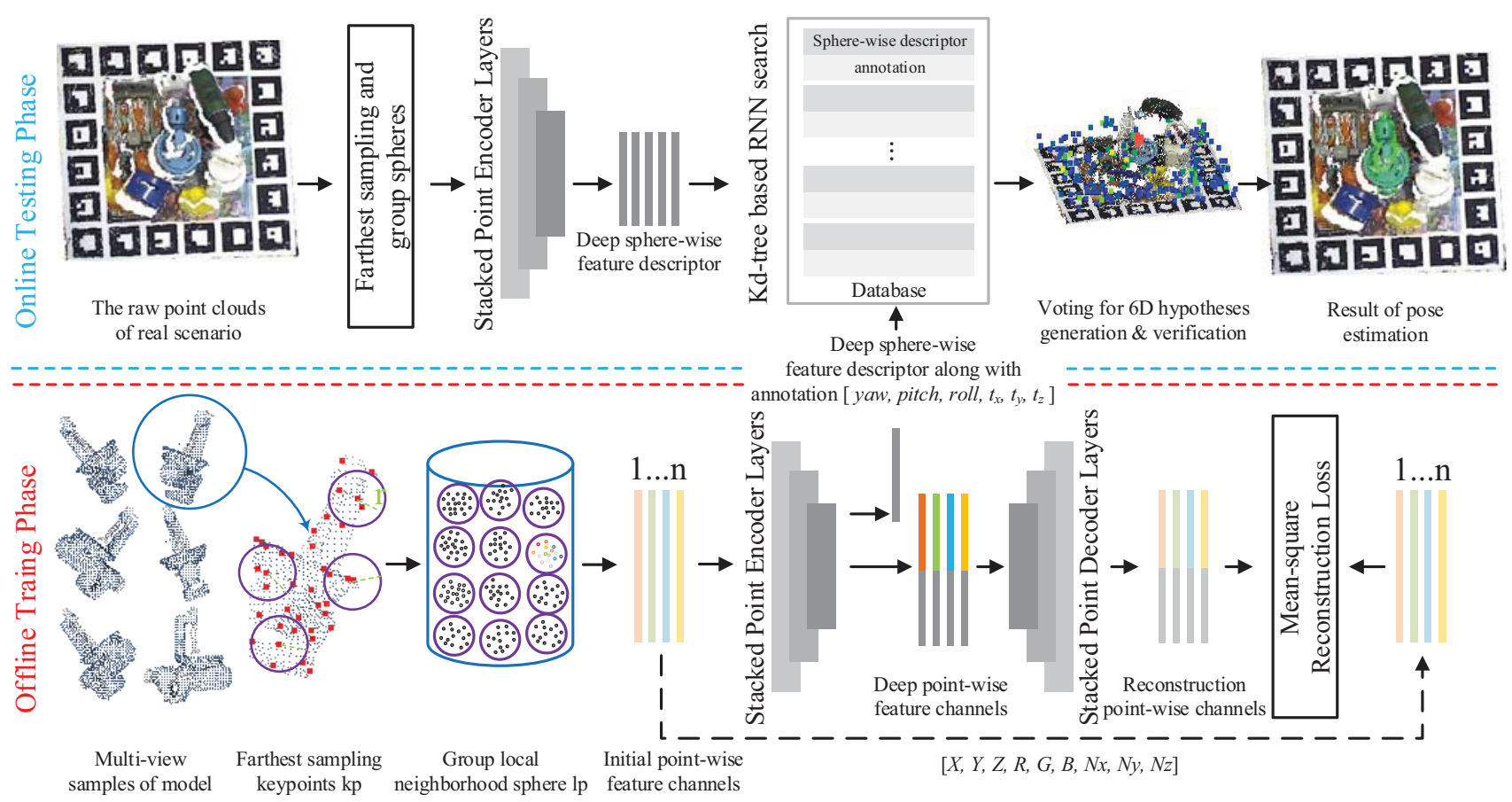

Figure 2: The framework of our 3D object recognition and 6-DOF pose estimation method. 1) In the offline training phase, the stacked point feature encoder is trained first and then generate feature database of all keypoints, which are sampled from synthetic point clouds of multiple model views. 2) In the online testing phase, the features from the unknown real scene are matched among the database by using the K-D tree searching method. Afterwards, the matching results are achieved by using the hypothesis \& verification strategy.

\subsection{The Offline Training Network}

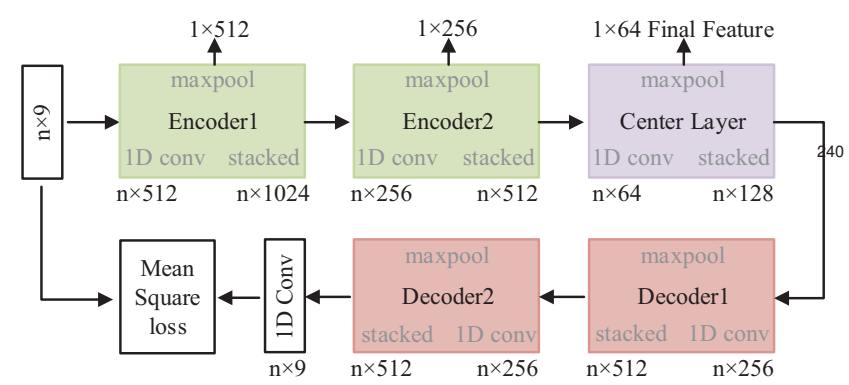

Figure 5: The complete framework and parameter configuration of the Autoencoder. It has three stacked encoder layers and three symmetric decoder layers. The center layer shared between the encoder and decoder. The output of the maxpool in the center layer is used as the final sphere-wise features. The meansquared error (MSE) is used as the reconstruction loss.

For the offline training, an unsupervised feature encoderdecoder network is presented as shown in Fig. 5. The decoders have the same structure to the encoders, which are used to reconstruct the input point clouds features. Several papers have proved that the features extracted by unsupervised reconstruction have effective performances $[26,27,28]$. In our case, the network has three stacked encoder layers and three symmetric decoder layers. The complete network structure and parameter configuration are show in Fig. 5, where the center layer is shared by the encoders and decoders. The output features of the maxpool in the center layer are used as the final sphere235 wise features (here the feature dimension is 64). Each encoder layer is composed of a 1D Convolution Layer, a maxpool layer and a stacked feature layer. The mean-squared error (MSE) is used as the reconstruction loss. For a visual impression of the reconstruction quality, the results of two random sampled spheres from testing scene are shown in Fig. 6, where both the reconstruction results of the $[X Y Z R G B]$ and the $\left[N_{x} N_{y} N_{z}\right]$ are shown almost similar. The $\left[N_{x} N_{y} N_{z}\right]$ shown is converted to the corresponding values within HSV color space.

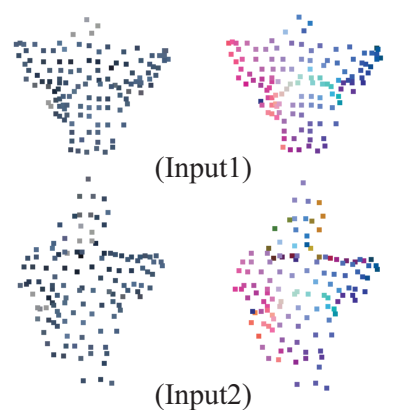

[XYZRGB] [NxNyNz]

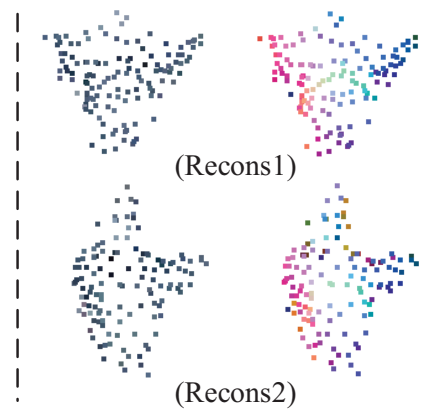

[XYZRGB]

$[\mathrm{NxNyNz}]$
Figure 6: The visual impression of the reconstruction quality for two sampled spheres from testing scene, where both the reconstruction results of the $[X Y Z R G B]$ and the normal $\left[N_{x} N_{y} N_{z}\right]$ shown are almost similar. The normal shown is converted to HSV color.

After the completion of the training, the features of all 3D 


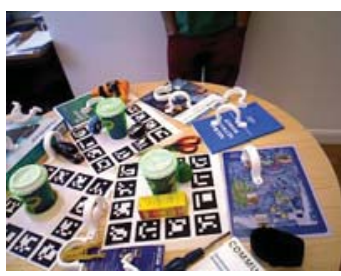

Recognition Results

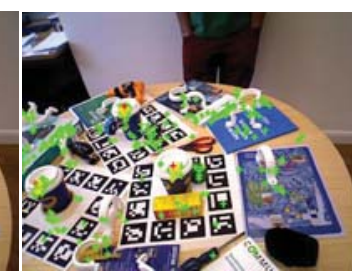

$\tau=0.7$

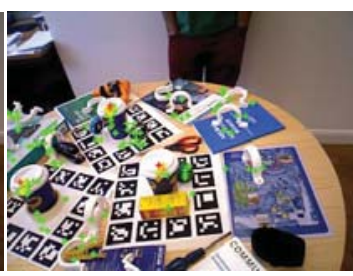

$\tau=0.8$

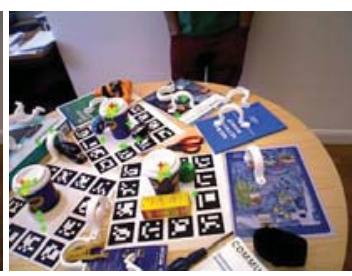

$\tau=0.9$

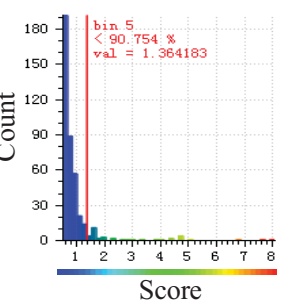

Score histogram

Figure 7: 6D cast voting space with different $\tau$ values. The projected voting centroids are colored following their voting score, which is mapped by an HSV colormap. The Saturation (S) and Value (V) are fixed to maximum and mapping the score to the range of Hue $(250 \rightarrow 360)$ corresponding to (blue $\rightarrow$ red). It can be seen clearly that with the increase of $\tau$, most false candidates are filtered out and the true candidates around the object centroid with a higher score is remained.

point clouds spheres $y$ sampled from synthetic model views are used to create a feature database, where each feature holds a local 6D pose annotation $\left[y a w\right.$, pitch, roll $\left., t_{x}, t_{y}, t_{z}\right] \in \mathbb{R}^{6}$. In n 200 our case, the annotation [yaw, pitch, roll] represents the pose transformation of each model view under the model's coordinate frame and the annotation $\left[t_{x}, t_{y}, t_{z}\right]$ is the offset from the sphere center $(x, y, z)$ to the model center $(0,0,0)$, where $\left(t_{x}=0-x, t_{y}=0-y, t_{z}=0-z\right)$. In this case, the model's coordinate frame is built on the model center.

\subsection{The Online Hypotheses Generation and Verification}

In this subsection, we intend to generate hypotheses of the candidate 6-DOF pose and refine them. For a given testing scene, the features of all 3D point clouds spheres sampled from the scene are exploited to find the pairwise correspondences from the feature database for 6-DOF pose estimation. Since pe size of the training feature database is huge to cover all sampling views of the object model, the K-D Tree searching method is used to search the optimal correspondences for each spherewise feature efficiently. During testing, we sample the keypoint $s=\left(s_{x}, s_{y}, s_{z}\right)$ with associated sphere $x$ from unknown scene

265 first and then compute its feature $f(x)$ and search the nearest spheres $y_{1}, \ldots, y_{m}$ from database. Each neighbor casts a global vote $v(s, y) \Rightarrow\left(t_{x}+s_{x}, t_{y}+s_{y}, t_{z}+s_{z}\right.$, yaw, pitch, roll $)$ with an associated weight as $w(v)=e^{-\|f(x)-f(y)\|}$ depending on the feature distance. This method is flexible enough to alter

270 the number of possible vote candidates by tuning the search radius $R$, and the searched candidates will only be voted if they hold a similar feature distance. This reduces the impact of noise sensitivity on the method and is more easily constrained by the number of votes. For different objects, the value of $R$ needs

275 to be adjusted manually according to the actual feature output. We adjust this value by observing and sampling several most similar feature distances.

Due to noise sensitivity and feature ambiguity, the valid voting candidates can lead to a crowded voting space,

280 which requires further refinement to make it computationally feasible. For the crowed 6-DOF voting space $\left[\right.$ yaw, pitch, roll, $\left.t_{x}, t_{y}, t_{z}\right]$, we first group it into equal voxel grids and add cumulative candidate weight $w(v)$ to each grid. Then, by computing the weight histogram, a dynamic threshold $285 \tau \in[0,1]$ is designed to reject $90 \%$ grids (in our case, $\tau=0.9$ ) with low weight. As $\tau$ increases, more false candidates are filtered out. The filter results of different $\tau$ are shown in Fig. 7. 300
For the remaining candidates, each is refined by the Iterative Closest Point (ICP) to refine the transformation and calculate a matching score $\varepsilon$, where $\varepsilon>0.8$ (in our case) means the overlap ratio between the object model and the scene surface. The non-maximum suppression is followed to find the local maximums depending on the voting weight, where each generates the final hypotheses $\left[R_{c}, T_{c}\right]$.

\section{Experiments}

In this section, we compare our method with several representative 3D object recognition methods, such as LineMod [21], SSD-6D [29], AE-HF [26], Spin image [15]. The experiments are evaluated on four publicly available datasets (the LC-HF dataset [25], the LineMod dataset [21], the AE-HF binpicking dataset [26]) and the UWA dataset [20], which contain multiple objects with various interferences, e.g., occlusion, illumination change, cluttered background and no-colors. For the evaluation metric, we first adopt the F1-score defined in LC-HF [25]. The estimation is deemed correct if the mean distance $m$ between the true pose $[R, T]$ of model $\mathcal{M}$ vertices and those estimated given the pose $\left[R_{c}, T_{c}\right]$ is less than $\lambda$ (here is $15 \%$ ) of the objects diameter [25]. Secondly, we adopt the Average of the 3D distance (ADD) metric defined in [21]. We take a pose estimate to be correct if the mean distance $m$ is less than $\lambda$ (here is 10\%) of the object diameter [21]. Specifically, for rotationally symmetric objects, the mean distance is computed as Eq. 1:

$$
m=\operatorname{avg} \sum_{x \in M} \min _{M}\left\|(R x+T)-\left(R_{c} x+T_{c}\right)\right\| .
$$

Thirdly, we adopt the Recognition rate under different Occlusion rate defined in [20]. The occlusion rate is defined as Eq. 2:

$$
\text { occlution }=1-\frac{\text { model visible surface area in scene }}{\text { total model surface area }} .
$$

\subsection{Results On the LC-HF Dataset [25][27]}

This dataset [25][27] contains 6 objects and each testing image has 2-3 same targets, which are placed on a cluttered round table. Each target is associated with the 3D mesh model and assigned a ground-truth $[R, T]$ matrix. 


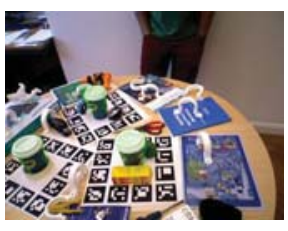

(A1) coffee

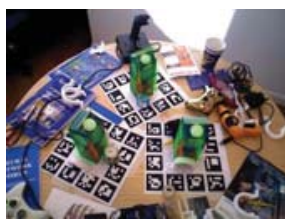

(D1) juice

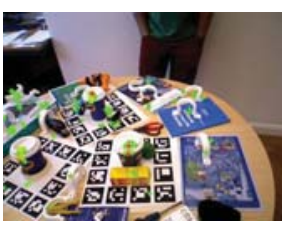

(A2) coffee

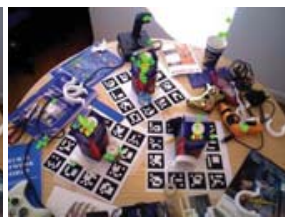

(D2) juice

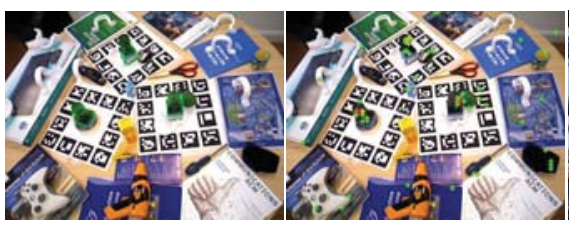

(B1) camera

(B2) camera

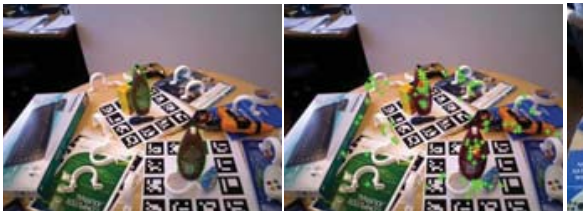

(E1) shampoo

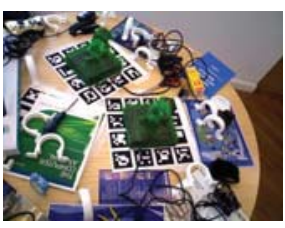

(C1) joystick

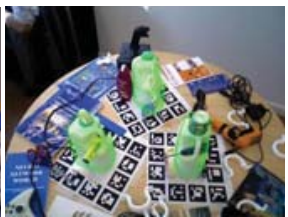

(F1) milk

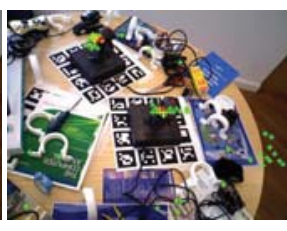

(C2) joystick

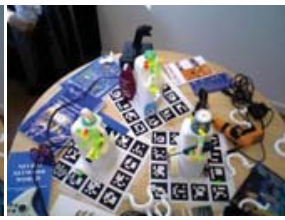

(F2) milk

Figure 8: Some demo results and the voting maps of our method on the LC-HF dataset [25][27], where the recognition results are shown as the green transformed model overlaid the estimated location (A1-F1). Areas with the high voting score are also clearly clustered in the object centroid (A2-F2).

Table 1: The statistic results of average F1-score of the re-annotated LC-HF datasets [25][27] in comparison with LineMod [21], LC-HF [25], ConvAE [27], VoxelAE [28] and SSD-6D [29]. The best results and the second best results are represented with red and blue fonts.

\begin{tabular}{lcccccc}
\hline \hline Objects & {$[21]$} & {$[25]$} & {$[27]$} & {$[28]$} & {$[29]$} & OURS \\
\hline Coffee & 0.942 & 0.891 & 0.972 & 0.977 & 0.983 & 0.996 \\
Shampoo & 0.922 & 0.792 & 0.910 & 0.857 & 0.892 & 0.931 \\
Joystick & 0.846 & 0.549 & 0.892 & 0.739 & 0.997 & 0.958 \\
Camera & 0.589 & 0.394 & 0.383 & 0.681 & 0.741 & 0.949 \\
Juice & 0.595 & 0.883 & 0.866 & 0.866 & 0.919 & 0.970 \\
Milk & 0.558 & 0.397 & 0.463 & 0.493 & 0.780 & 0.831 \\
\hline Average & 0.740 & 0.651 & 0.747 & 0.768 & 0.885 & 0.939 \\
\hline \hline
\end{tabular}

The statistic recognition results are shown in Tab. 1, where the overall average F1-score of our method is $93.9 \%$, in comparison with LineMod (74.0\%) [21], LC-HF (65.1\%) [25], ConvAE (74.7\%) [27], VoxelAE (76.8\%) [28] and SSD-6D $(88.5 \%)$ [29] respectively. Here, [29] counts a detection to be correct when the IoU score of a predicted bounding box with the groundtruth box is higher than 0.5 . It is evident that LineMOD fares very well on most sequences with low occlu-345 sion (e.g., coffee, shampoo and joystick). It only shows problem where objects sometimes are partially visible (e.g., milk) or where the objects are confused by the background (e.g., camera and juice). LC-HF improves the inherent robustness to foreground occlusions by using patch representation, but its overall $1_{350}$ performance is not fully exploited due to the complex parameter adjustment of hough voting strategy. ConvAE improves the overall performance by using deep RGBD patch representation and combining simple and valid voting strategy. Due to the loss of geometric information in the process of projection from $3 \mathrm{D}_{355}$ to $2 \mathrm{D}$ for RGBD data, it decreases the precision by the cluttered environment. Although VoxelAE deals 3D points, the process of voxelization also leads to the loss of geometry information and introduces unnecessary memory expenditure. SSD-6D is a supervised CNN-based method, which requires a lot of scene 360 distribution data and predicts the object 2D bounding box and a rough estimate of the objects orientation in RGB image. The final 6-DOF estimation is calculated by several stages of refinement and verification. This process will magnify the error of $2 \mathrm{D}$ prediction and then affect the 6-DOF pose estimation. In contrast to most existing methods, ours deals 3D points without any data conversion (e.g., voxelization), which makes full use of geometric information and does not dataset specific.

Ours also give rise to a good result for the camera model and the joystick model, where the camera model is small in size and looks similar to the background and the joystick model has thin and thick part. Especially for the milk model, while this model is texture-less, smooth-surface and contains other distracting objects on it, ours shows better results. It is evident that the learned features can handle various object appearance. Fig. 8 demonstrates our recognition results on the LC$\mathrm{HF}$ dataset, where ours can accurately estimate the objects pose with amounts of clutter. We use the metric Eq. 1 when evaluating the pose accuracy for the rotationally invariant objects, coffee, shampoo, camera and juice.

\subsection{Results On the LineMod Datasets [21]}

This dataset [21] contains 15 objects and each testing image has only one target, which is placed on a desk with heavy amounts of occlusion and clutter. Each object is associated with the 3D mesh model and assigned a ground-truth $[R, T]$ matrix in more than $1 \mathrm{k}$ testing images. Since the mesh models of the bowl and the cup are missing, we test the other 13 models as well as [25]. The statistic recognition results of average F1score and ADD metric are shown in Tab. 2.

Firstly, we also give the statistic recognition results of average F1-score in comparison with LineMod [21], LC-HF [25], ConvAE [27] and SSD-6D [29] respectively, where the average F1-score of our proposed method is $93.4 \%$ outperforming the state-of-the-arts, e.g., the second best one ConvAE (92.88\%), the third best one SSD-6D $(88.50 \%)$ and so on. Here, [29] counts a detection to be correct when the IoU score of a predicted bounding box with the groundtruth box is higher than 0.5 . Specially, ours yields the best results for 3 out of all 13 


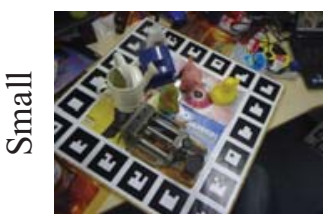

(A1) ape

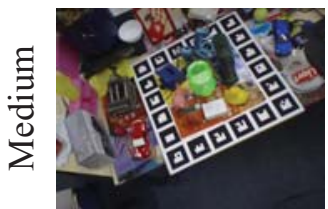

(D1) can

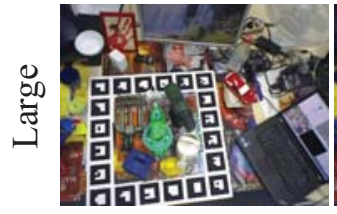

(G1) bvise

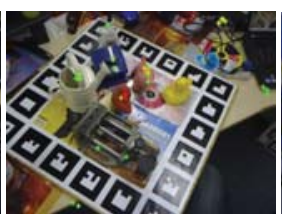

(A2) ape

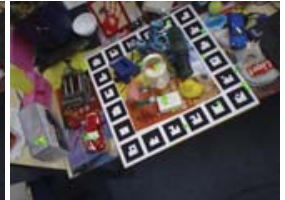

(D2) can

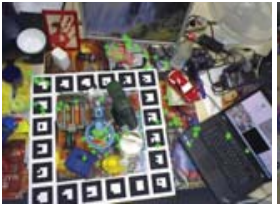

(G2) bvise

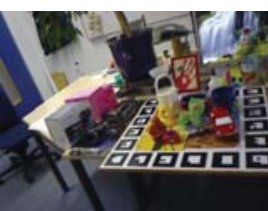

(B1) cat

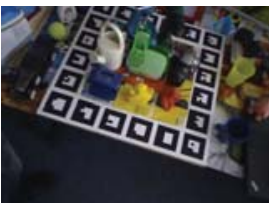

(E1) phone

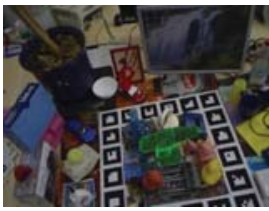

(H1) driller

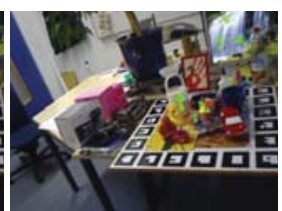

(B2) cat

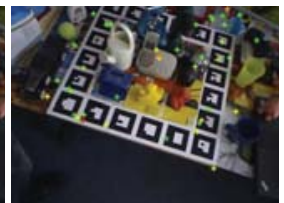

(E2) phone

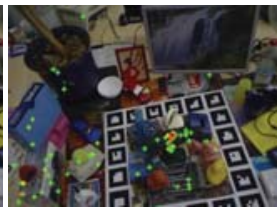

(H2) driller

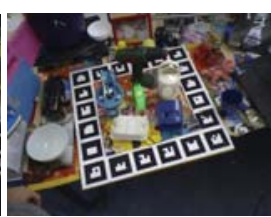

(C1) glue

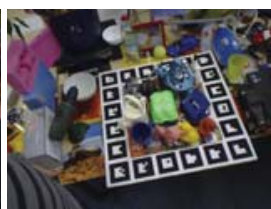

(F1) eggbox

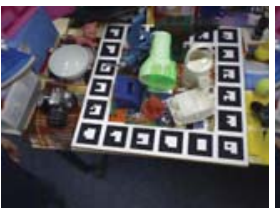

(I1) lamp

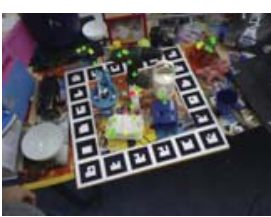

(C2) glue

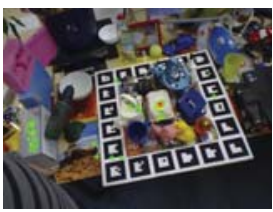

(F2) eggbox

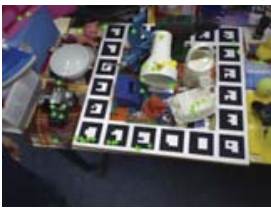

(I2) lamp

Figure 9: Some demo results and the voting maps of our method on the LineMod dataset [21], where the recognition results are shown as the green transformed model overlaid the estimated location (A1-I1). We roughly divided the objects into three scales: the small size (top row), the medium size (middle row) and the large size (bottom row). Areas with the high voting score are also clearly clustered in the object centroid (A2-I2).

Table 2: The statistic results of average F1-score and ADD metric on the LineMod dataset [21] in comparison with LineMod [21], LC-HF [25], ConvAE [27], SSD-6D [29], BB8 [30] and Seamless [31]. The best results and the second best results are expressed in red and blue fonts. The ADD metric of Seamless [31] is acquired without refinement and for reference only.

\begin{tabular}{l|lllll|l|l|l}
\hline \hline Metric & \multicolumn{7}{|c}{ F1-Score $(\lambda=0.15)$} & ADD $(\lambda=0.1)$ \\
\hline \hline Objects $\mid[21]$ & {$[25]$} & {$[27]$} & {$[29]$} & OURS $\mid$ & {$[31]$} & {$[30]$} & OURS \\
\hline ape & 53.3 & 85.5 & 98.1 & 76.3 & 93.5 & 21.62 & 40.4 & 68.6 \\
bvise & 84.6 & 96.1 & 94.8 & 97.1 & 95.2 & 81.80 & 91.8 & 78.9 \\
cam & 64.0 & 71.8 & 93.4 & 92.2 & 96.3 & 36.57 & 55.7 & 87.2 \\
can & 51.2 & 70.9 & 82.6 & 93.1 & 94.3 & 68.80 & 64.1 & 83.0 \\
cat & 65.6 & 88.8 & 98.1 & 89.3 & 94.8 & 41.82 & 62.6 & 84.4 \\
driller & 69.1 & 90.5 & 96.5 & 97.8 & 93.2 & 63.51 & 74.4 & 80.5 \\
duck & 58.0 & 90.7 & 97.9 & 80.0 & 96.2 & 27.23 & 44.3 & 81.3 \\
eggb & 86.0 & 74.0 & 100 & 93.6 & 96.0 & 69.58 & 57.8 & 84.4 \\
glue & 43.8 & 67.8 & 74.1 & 76.3 & 88.2 & 80.02 & 41.2 & 62.8 \\
holep & 51.6 & 87.5 & 97.9 & 71.6 & 90.8 & 42.63 & 67.2 & 77.8 \\
iron & 68.3 & 73.5 & 91.0 & 98.2 & 92.3 & 74.97 & 84.7 & 81.3 \\
lamp & 67.5 & 92.1 & 98.2 & 93.0 & 95.7 & 71.11 & 76.5 & 85.8 \\
phone & 56.3 & 72.8 & 84.9 & 92.4 & 88.4 & 47.74 & 54.0 & 74.5 \\
\hline Average & 83.44 & 81.69 & 92.88 & 88.50 & 93.40 & 55.95 & 62.7 & 79.3 \\
\hline
\end{tabular}

models and all the others are getting the second best results. Although the results of most models are not the best, ours are the most balanced by integrating 3D geometric information.

Secondly, we give the statistic recognition results of AD$\mathrm{D}$ metric, where the ADD metric of our proposed method is $79.3 \%$, in comparison with more CNN-based method, BB8 $(62.7 \%)$ [30] and Seamless (55.95\% without refinement for reference only) [31]. BB8 is made of one CNN to coarsely seg-390 370 ment the object and another to predict the projections of the objects 3D bounding box given the segmentation in 2D image, which are then used to compute the $6 \mathrm{D}$ pose using a $\mathrm{PnP}[30]$ algorithm and further pose refinement. Unlike BB8 methods, which require multi-stage of processing, Seamless is a singleshot method that takes the image as input and directly detects the $2 \mathrm{D}$ projections of the $3 \mathrm{D}$ bounding box vertices. The objects $6 \mathrm{D}$ pose is then estimated using the $\mathrm{PnP}$ algorithm without any refinement. For these methods, the main problem is to predict in the 2D image space, and then get the 6-DOF estimation of the objects in the $3 \mathrm{D}$ space by spatial mapping. The error of $2 \mathrm{D}$ prediction is further magnified in the process of spatial mapping. In contrast to these methods, the overall ADD metric of ours shows better results.

Fig. 9 demonstrates our recognition results on the LineMod dataset, where ours can accurately estimate the objects pose with heavy amounts of occlusion, scale change and clutter. We use the metric Eq. 1 when evaluating the pose accuracy for the rotationally invariant objects, glue, eggbox as well as [21].

Table 3: The statistic results of average F1-score on the AE-HF bin-picking dataset [26]. The best results and the second best results are expressed in red and blue fonts.

\begin{tabular}{lccc}
\hline \hline Objects & LC-HF[25] & AE-HF [26] & OURS \\
\hline CoffeeCup & 0.314 & 0.361 & 0.469 \\
\hline Juice & 0.248 & 0.290 & 0.412 \\
\hline
\end{tabular}

\subsection{Results On the AE-HF Bin-picking Datasets [26]}

This dataset [26] constructs two bin-picking scenarios, where each contains multiple same targets, 16 for bin-coffee scenario and 5 for bin-juice scenario. Different from the two household datasets LC-HF datset [25] and LineMod dataset [21] with ob- 


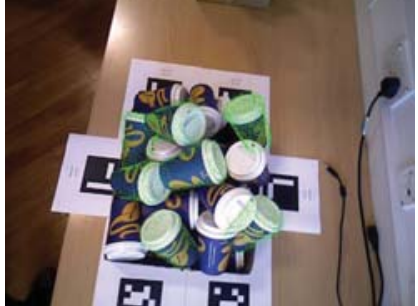

(A1) bin-coffee

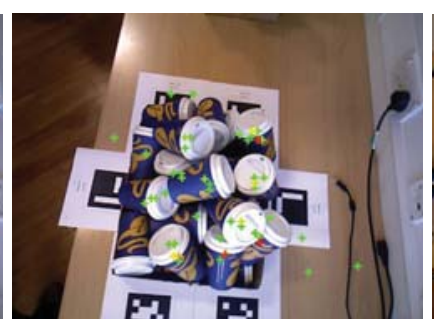

(A2) bin-coffee

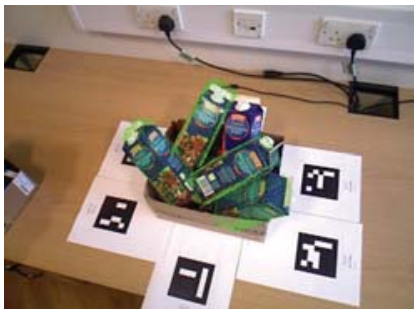

(B1) bin-juice

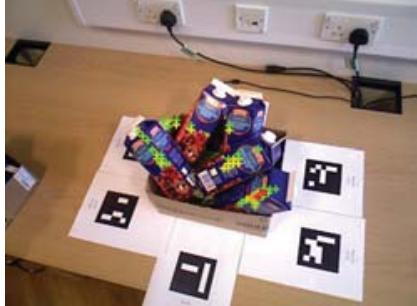

(B2) bin-juice

Figure 10: Some demo results and the voting maps of our method on the AE-HF bin-picking dataset [26], where the recognition results are shown as the green transformed model overlaid the estimated location (A1, B1). As can be seen that ours can accurately recognize the objects pose even with heavy amounts of self-occlusion (A2, B2).

jects placed separately, the serious aliasing and self-occlusion 395

The statistic recognition results of average F1-Score are shown in Tab. 3, where our method outperforms LC-HF [25] and AE-HF [26] with about $30 \%$ improvement. For the most existing RGB-D patch-based methods (e.g., LC-HF, AE-HF),

400 the features usually contain more interferences due to the local 425 patches that inevitably cover part of the background, as shown in Fig. 11 (A), where the red part is the background. In contrast, our method extracts features from the local point clouds spheres, which contains relatively little interference because of cal sphere contains only the surface of the target without any background. Fig. 10 demonstrates our recognition results on the AE-HF bin-picking dataset, where the targets have accurate voting centers. As a result, ours are able to accurately recognize 410 the object pose even with heavy amounts of self-occlusion.

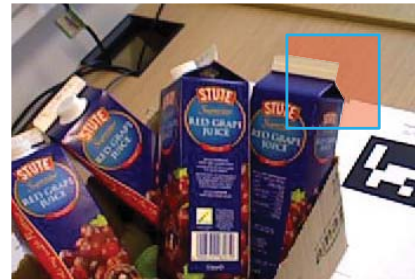

(A) RGB-D Patch

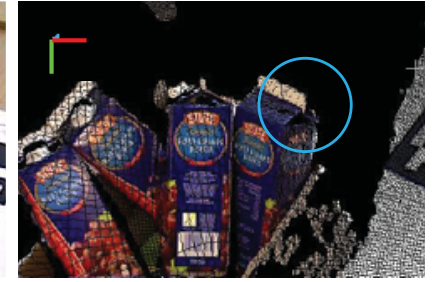

(B) Point Clouds Sphere
Figure 11: The demonstration of the local RGB-D patch and the local point clouds sphere. (A) The RGB-D patch contains part of the background. (B) The point clouds sphere contains only the surface of the target without any background.

\subsection{Results On the UWA Datasets}

The UWA dataset [20] contains 5 objects and 50 real test-430 ing scenes, where each scene is scanned by a Minolta VIVID 910 laser scanner by placing 4-5 different models in the scene without color information. Exclude the Rhino model due to it contains large holes and cannot be recognized well, other objects are associated with the $3 \mathrm{D}$ mesh model and assigned $\mathrm{a}_{435}$ ground-truth $[R, T]$ matrix.
Specifically, we use $\left[X, Y, Z, N_{x}, N_{y}, N_{z}\right]$ without RGB information as the only input of the feature encoders. The statistic recognition results are shown in Tab. 4 , when the object occlusion rate is between 0 and $84 \%$, the overall average recognition rate of our method is the second best $97.7 \%$, in comparison with Tensor (96.6\%) [15], Spin image (87.8\%) [15], EM (97.5\%) [16] and RoPS (98.8\%) [17] respectively.

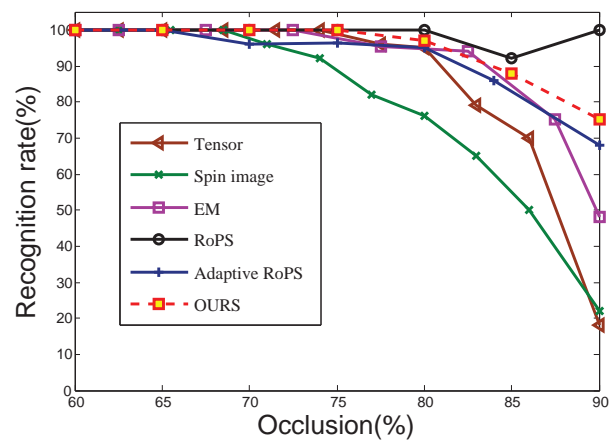

Figure 14: The recognition rate against different occlusion on the UWA dataset without Rhino model.

Table 4: The statistic results of average recognition rate on the UWA dataset [20] without Rhino model, where the the occlusion rate is between 0 and $84 \%$. The best results and the second best results are expressed in red and blue fonts.

\begin{tabular}{lccccc}
\hline \hline \multicolumn{5}{c}{ The occlusion rate is between 0 and 84\% } \\
\hline Methods & Tensor [15] & Spin image[15] & EM [16] & RoPS [17] & OURS \\
\hline Avg & 96.6 & 87.8 & 97.5 & 98.8 & 97.7 \\
\hline
\end{tabular}

Although our method is designed to be better at dealing with scenes with color information, it is still applicable for such no-color dataset. Compared with other special methods, ours achieves $97.7 \%$ recognition rate when the occlusion rate is between 0 and $84 \%$, which is close to the optimal RoPS method. As shown in Fig. 14, when the occlusion rate exceeds $84 \%$, the recognition rate of ours is still better than most of the state-ofthe-arts. In addition, we manually patched the Rhino model as shown in Fig. 12 and labeled all 23 scenes containing Rhino using ICP method. The overall average recognition rate of our method is $92.3 \%$, when the occlusion rate of Rhino model 


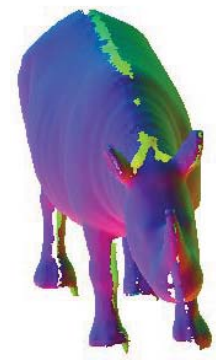

(A) Rhino model with holes

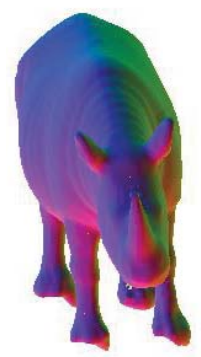

(B) Rhino model after patched

Figure 12: Rhino model patched with Geomagic Studio. We convert the normal to HSV color space for visualization.

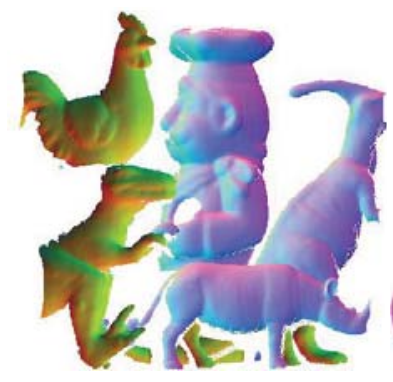

(A1) The first sample scene

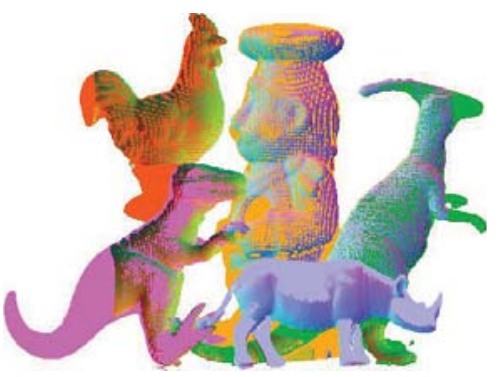

(A2) Our recognition result

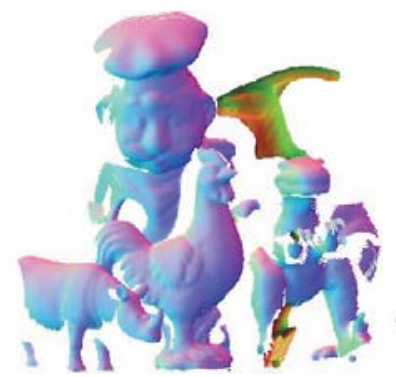

(B1) The second sample scene

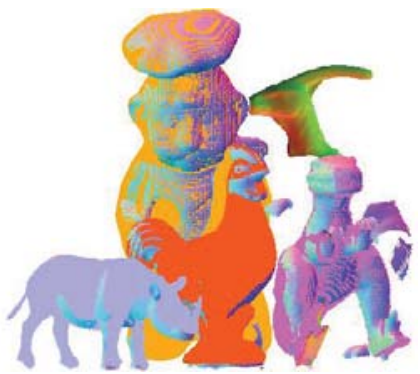

(B2) Our recognition result

Figure 13: Two sample scenes and our recognition results on the UWA dataset, the correctly recognized objects are covered by their $3 \mathrm{D}$ models. We convert the normal to HSV color space for visualization.

is between 0 and $74 \%$. The overall average recognition rate is $52.2 \%$ in the whole occlusion range. Fig. 13 demonstrates our 455 recognition results on two sample scenes on the UWA Dataset, where ours can also accurately recognize objects without color.

\subsection{Results on the Average Running Time}

We present the average time consumption of our method on the LC-HF dataset [25] and the comparison with ConvAE [27]. As shown in Tab. 5, we record the corresponding time of varpotheses generation and Refinement. The total average running time for our method is $774 \mathrm{~ms}$, which is close to the $670 \mathrm{~ms}$ usage of ConvAE. Specifically, we use different platforms for different phases and record the time separately.

Table 5: Comparisons of the average runtime of ours and ConvAE [27] on the LC-HF dataset [25].

\begin{tabular}{l|c|c}
\hline \hline Stage & ConvAE $(\mathrm{ms})$ & OURS $(\mathrm{ms})$ \\
\hline \hline Data Sampling & 0.03 & 12.5 \\
Feature Extraction & 477.3 & 47.4 \\
Hypothesis Generation & 63 & 186.2 \\
Refinement & 130.5 & 528.4 \\
\hline Online testing & 670.8 & 774.5 \\
\hline \hline
\end{tabular}

In our case, it contains data sampling (keypoints $k_{p}$ and local points $l_{p}$ sampling) and spheres grouping. We use GPU for parallel acceleration, which can sample and group the raw point clouds of more than $30 \mathrm{~K}$ into local spheres of $4 \mathrm{~K}$ with about $12 \mathrm{~ms}$. The feature extraction use about $47 \mathrm{~ms}$, which is less than ConvAE $(477 \mathrm{~ms})$. We implement this phase on the Tensorflow framework based on Pointnet model [33] with a NVIDIA TITAN XP (12GB RAM). The Hypotheses generation and Refinement consume more time than ConvAE due to operations on point clouds directly. Both the Hypotheses generation and Refinement are executed on a standard PC with a general Intel CPU (i5-3470) at 3.20GHz, 16GB RAM.

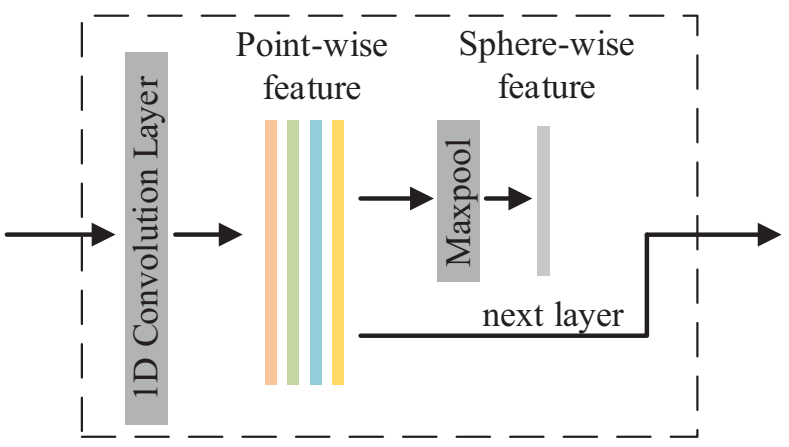

Non-stacked Point Feature Encoder Layer

Figure 15: The architecture of the Non-stacked Point Feature Encoder Layer, which generates the features without stacked operations.

\subsection{Comparisons on the Different Network Architecture}

In order to justify the effectiveness of the stacked point feature encoder, we compare it with another architecture on the 
AE-HF bin-picking dataset [26].

As shown in Fig. 15, we extract the sphere-wise features and point-wise features for this architecture without stacked operations. Given a normalized sphere with initial point-wise features, we transform it through the 1D convolution layer into a gregate information from the new point-wise features to spherewise features. The statistic recognition results of average F1Score are shown in Fig. 16, where we compare two architectures with different number of encoder layers. We can see intuitively that for both the two different architectures, by increasing the number of encoder layers from $1 \sim 3$ (include the center layer), the recognition results increase obviously. By fixing the number of the encoder layers, the recognition result of the stacked point feature encoder in our case is more effective than the non-stacked point feature encoder, which can be attributed to the fact that the stacked operation augments the local sphere features by concatenating the locally aggregated features layer by layer.

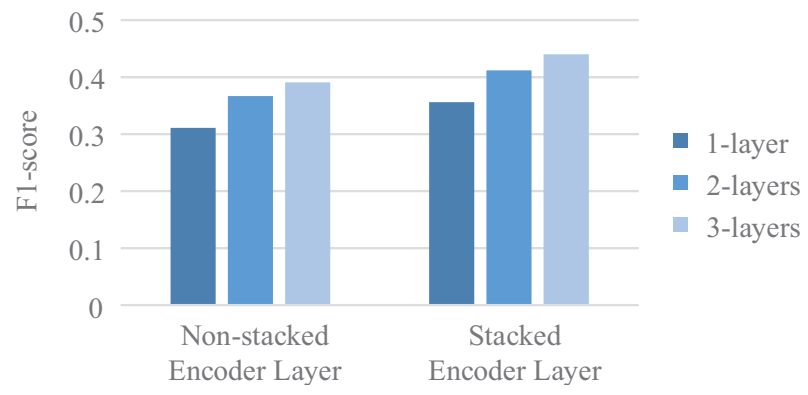

Figure 16: Comparisons on the performance of the two architecture with the different number of encoder layers.

\subsection{Comparisons on the Different Sampling Methods}

One of the most important processes for 3D point clouds in object recognition scenarios is to sample the input point clouds $(\sim 30 \mathrm{~K})$ to decrease the memory/efficiency burden on the computing platform. The most commonly used sampling methods for point clouds are Voxel-based Uniform Sampling (VS) [45] and Random Sampling (RS) [44]. However, the VS method is unable to limit the sampling points number, which is not suitable for our network structure, and ours needs a fixed input size. The RS method is suitable for our network, but the sampling points cannot fully cover the surface of the input dabecause of its inherent randomness during sampling. To this end, we adopt the Farthest Point Sampling (FPS) [43] method and present the comparison results with RS on the AE-HF bin-520 picking dataset [26] as shown in Fig. 17. By fixing the keypoints number $(k p=4096)$ and the local points number $(l p=$ 256), we can see that the recognition result of the FPS is more effective than the RS method due to the FPS can fully cover the surface of the input point clouds.

In addition, we present the recognition results with different FPS sampling number of the $k p$ and $l p$ as shown in Fig. 18,

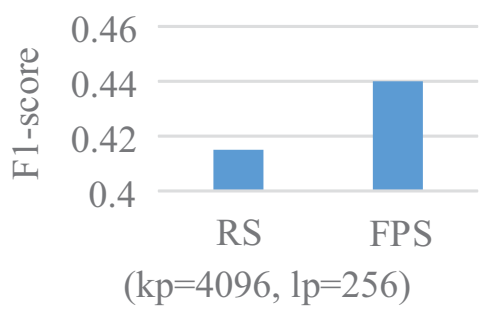

Figure 17: Compare the performance of RS [44] and FPS [43].

$k p$ that is designed to increase from 1024 to 4096 . By fixing the $k p$ and increasing the $l p$, the average F1-score can also be improved. But when the $l p$ exceeds 128 (in our case), it offers a slight improvement and comes at the expense of additional computational time. This is because the increase of $k p$ augments the coverage of the target surface, and the increase of the $l p$ can also enhance the expression of the local features, but when exceeding a threshold, it can fully express the local surface without more.

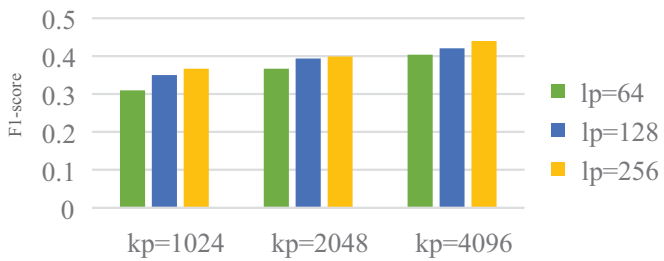

Figure 18: Comparisons on the performances of different sampling size of keypoints $k_{p}$ and sampling size of local points $l_{p}$.

\subsection{Comparisons on the Different Support Radius $r$}

The support radius $r$ determines the range of the local sphere, which contains the local surface points for feature extraction. We present the recognition results of different size of the support radius $r$ on the AE-HF bin-picking dataset [26].

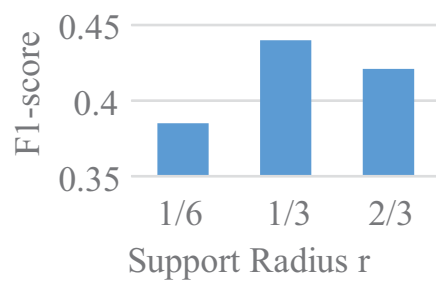

Figure 19: Comparisons on the performance of different support radius $r$.

We varied the size of the support radius $r$ to $\frac{1}{6}, \frac{1}{3}$ and $\frac{2}{3}$, which means the proportion of the shortest object dimensions. As shown in Fig. 19, the $\frac{1}{3}$ shows the best performance. It also shows that an increase in the support radius $r$ significantly improves the accuracy $\left(\frac{1}{6} \sim \frac{1}{3}\right)$, while on the other hand, an excessive increase $\left(\frac{2}{3}\right)$ of the $r$ offers a slight decrease. This is because the appropriate support radius $r$ is sufficient to express local features; instead, an oversize $r$ will incur background interference. 


\section{Conclusions}

530

In this paper, a deep stacked point feature is designed for 3D point clouds representation, which is able to preserve the original geometric information of objects to the greatest extent. We $\mathrm{W}_{595}$ scrap the handcrafted feature engineering for $3 \mathrm{D}$ point cloud$\mathrm{s}$ and propose a simple but effective unsupervised feature enpoint clouds spheres, which avoids the geometric information 600 loss and reduces the computational costs. The feature extraction is enabled within a local neighborhood sphere, by stacking the locally sphere-wise aggregated feature on point-wise feais trained first and then generate a feature database of all keypoints, which are sampled from synthetic model views. During online testing, a number of scene features, which are sampled by farthest sampling method, match against the database of syn-610 sequently filtered to refine hypotheses. The proposed method is evaluated on four datasets and the results prove that ours can generalize well to multiple scenarios and deliver comparable615 or even superior performance than the state-of-the-arts. In the future work, we intend to extend the current pipeline to a supervised end-to-end network, which operates on pure point clouds and directly predicts the 6-DOF pose in 3D space.

\section{References}

[1] S. A. A. Shah, M. Bennamoun, F. Boussaid, Keypoints-based surface representation for $3 \mathrm{~d}$ modeling and $3 \mathrm{~d}$ object recognition, Pattern Recognition 64 (2017) 29-38.

[2] U. Asif, M. Bennamoun, F. A. Sohel, Rgb-d object recognition and grasp detection using hierarchical cascaded forests, IEEE Transactions ${ }_{630}$ on Robotics 33 (3) (2017) 547-564.

[3] M.-L. Torrente, S. Biasotti, B. Falcidieno, Recognition of feature curves on $3 \mathrm{~d}$ shapes using an algebraic approach to hough transforms, Pattern Recognition 73 (2018) 111-130.

[4] M. Wang, Y. Gao, K. Lu, Y. Rui, View-based discriminative probabilistic ${ }_{635}$ modeling for $3 \mathrm{~d}$ object retrieval and recognition, IEEE Transactions on Image Processing 22 (4) (2013) 1395-1407.

[5] X. Li, M. Fang, J. J. Zhang, J. Wu, Learning coupled classifiers with rgb images for rgb-d object recognition, Pattern Recognition 61 (2017) 433446.

[6] Y. Cong, G. Sun, J. Liu, H. Yu, J. Luo, User attribute discovery with missing labels, Pattern Recognition 73 (2018) 33-46.

[7] J. M. Morel, G. Yu, Asift: A new framework for fully affine invariant image comparison, SIAM journal on imaging sciences 2 (2) (2009) 438469.

[8] D. G. Lowe, Distinctive image features from scale-invariant keypoints, International journal of computer vision 60 (2) (2004) 91-110.

[9] H. Bay, A. Ess, T. Tuytelaars, L. Van Gool, Speeded-up robust features (surf), Computer vision and image understanding 110 (3) (2008) 346359.

[10] A. Collet, M. Martinez, S. S. Srinivasa, The moped framework: Object recognition and pose estimation for manipulation, International Journal of Robotics Research 30 (10) (2011) 1284-1306.

[11] J. Tang, S. Miller, A. Singh, P. Abbeel, A textured object recognition pipeline for color and depth image data, in: ICRA, 2012, pp. 3467-3474.

[12] T. Zhou, X. Jing, Surface-based detection and 6-dof pose estimation of 3-d objects in cluttered scenes, IEEE Transactions on Robotics PP (99) (2016) 1-15.

[13] A. E. Johnson, M. Hebert, Surface matching for object recognition in complex three-dimensional scenes, Image and Vision Computing 16 (9- ${ }_{660}$ 10) (1998) 635-651.

[14] Y. Guo, F. Sohel, M. Bennamoun, M. Lu, J. Wan, Trisi: A distinctive local surface descriptor for $3 \mathrm{~d}$ modeling and object recognition, in: GRAPP/IVAPP, 2015.

[15] A. S. Mian, M. Bennamoun, R. Owens, Three-dimensional model-based object recognition and segmentation in cluttered scenes, IEEE transactions on pattern analysis and machine intelligence 28 (10) (2006) 1584 1601.

[16] P. Bariya, J. Novatnack, G. Schwartz, K. Nishino, 3d geometric scale variability in range images: Features and descriptors, International journal of computer vision 99 (2) (2012) 232-255.

[17] Y. Guo, F. Sohel, M. Bennamoun, M. Lu, Wan, Rotational projection statistics for $3 \mathrm{~d}$ local surface description and object recognition, International journal of computer vision 105 (1) (2013) 63-86.

[18] Y. Guo, M. Bennamoun, F. Sohel, M. Lu, J. Wan, N. M. Kwok, A comprehensive performance evaluation of $3 \mathrm{~d}$ local feature descriptors, International journal of computer vision 116 (1) (2016) 66-89.

[19] Y. Cong, D. Tian, Y. Feng, B. Fan, H. Yu, Speedup 3-d texture-less objec$\mathrm{t}$ recognition against self-occlusion for intelligent manufacturing, IEEE transactions on cybernetics 48 (99) (2018) 1-11.

[20] A. Mian, M. Bennamoun, R. Owens, On the repeatability and quality of keypoints for local feature-based $3 \mathrm{~d}$ object retrieval from cluttered scenes, International Journal of Computer Vision 89 (2-3) (2010) 348-361.

[21] S. Hinterstoisser, V. Lepetit, S. Ilic, S. Holzer, G. Bradski, K. Konolige, N. Navab, Model based training, detection and pose estimation of textureless $3 \mathrm{~d}$ objects in heavily cluttered scenes, in: ACCV, 2012, pp. 548-562.

[22] S. Hinterstoisser, C. Cagniart, S. Ilic, P. Sturm, N. Navab, P. Fua, V. Lep etit, Gradient response maps for real-time detection of textureless object$\mathrm{s}$, IEEE transactions on pattern analysis and machine intelligence 34 (5) (2012) 876-888.

[23] R. Rioscabrera, T. Tuytelaars, Discriminatively trained templates for $3 \mathrm{~d}$ object detection: A real time scalable approach, in: ICCV, 2014, pp. 2048-2055.

[24] W. Kehl, F. Tombari, N. Navab, S. Ilic, V. Lepetit, Hashmod: A hashing method for scalable 3d object detection, in: BMVC, 2016.

[25] A. Tejani, D. Tang, R. Kouskouridas, T. K. Kim, Latent-class hough forests for $3 \mathrm{~d}$ object detection and pose estimation, in: ECCV, 2014, pp. $462-477$.

[26] A. Doumanoglou, R. Kouskouridas, S. Malassiotis, T. K. Kim, Recovering $6 \mathrm{~d}$ object pose and predicting next-best-view in the crowd, in: CVPR, 2016, pp. 3583-3592.

[27] W. Kehl, F. Milletari, F. Tombari, S. Ilic, N. Navab, Deep learning of local rgb-d patches for $3 \mathrm{~d}$ object detection and $6 \mathrm{~d}$ pose estimation, in: $\mathrm{ECCV}$, 2016, pp. 205-220.

[28] H. Liu, Y. Cong, S. Wang, H. Fan, D. Tian, Y. Tang, Deep learning of directional truncated signed distance function for robust $3 \mathrm{~d}$ object recognition, in: IROS, 2017, pp. 5934-5940.

[29] W. Kehl, F. Manhardt, F. Tombari, S. Ilic, N. Navab, Ssd-6d: Making rgb-based $3 \mathrm{~d}$ detection and $6 \mathrm{~d}$ pose estimation great again, in: CVPR, 2018 .

[30] M. Rad, V. Lepetit, Bb8: A scalable, accurate, robust to partial occlusion method for predicting the $3 \mathrm{~d}$ poses of challenging objects without using depth, in: ICCV, Vol. 1, 2017, p. 5 .

[31] B. Tekin, S. N. Sinha, P. Fua, Real-time seamless single shot 6d object pose prediction, in: CVPR, 2018.

[32] R. Q. Charles, H. Su, K. Mo, L. J. Guibas, Pointnet: Deep learning on point sets for $3 \mathrm{~d}$ classification and segmentation, in: CVPR, 2017, pp. 77-85.

[33] C. R. Qi, L. Yi, H. Su, L. J. Guibas, Pointnet++: Deep hierarchical feature learning on point sets in a metric space, in: NIPS, 2017, pp. 5099-5108.

[34] D. Chetverikov, D. Svirko, D. Stepanov, P. Krsek, The trimmed iterative closest point algorithm, in: ICPR, Vol. 3, 2002, pp. 545-548.

[35] R. Klokov, V. Lempitsky, Escape from cells: Deep kd-networks for the recognition of 3d point cloud models, in: ICCV, 2017, pp. 863-872.

[36] S. Song, J. Xiao, Sliding shapes for 3d object detection in depth images, in: ECCV, 2014, pp. 634-651.

[37] R. Girdhar, D. F. Fouhey, M. Rodriguez, A. Gupta, Learning a predictable and generative vector representation for objects, in: ECCV, 2016, pp. 484-499.

[38] S. Gupta, R. Girshick, P. Arbelez, J. Malik, Learning rich features from rgb-d images for object detection and segmentation, in: ECCV, Vol. 8695, 2014 , pp. 345-360. 
[39] S. Gupta, P. Arbelez, R. Girshick, J. Malik, Inferring 3d object pose in rgb-d images, ArXiv preprint arXiv:1502.04652.

[40] J. Leitner, A. W. Tow, N. Snderhauf, J. E. Dean, J. W. Durham, M. Cooper, M. Eich, C. Lehnert, R. Mangels, C. Mccool, The acrv picking benchmark: A robotic shelf picking benchmark to foster reproducible research, in: ICRA, 2017, pp. 4705-4712.

[41] A. Zeng, K. T. Yu, S. Song, D. Suo, E. Walker, A. Rodriguez, J. Xiao, Multi-view self-supervised deep learning for $6 \mathrm{~d}$ pose estimation in the amazon picking challenge, in: ICRA, 2017, pp. 1386-1383.

[42] V. Lepetit, F. Morenonoguer, P. Fua, Epnp: An accurate o(n) solution to the pnp problem, International Journal of Computer Vision 81 (2) (2009) $155-166$.

[43] C. Moenning, N. A. Dodgson, Fast marching farthest point sampling for implicit surfaces and point clouds, Computer Laboratory Technical Report 565 (2003) $1-12$

[44] J. S. Vitter, Random sampling with a reservoir, ACM Transactions on Mathematical Software 11 (1) (1985) 37-57.

[45] R. B. Rusu, S. Cousins, 3d is here: Point cloud library (pcl), in: ICRA, IEEE, 2011, pp. 1-4.

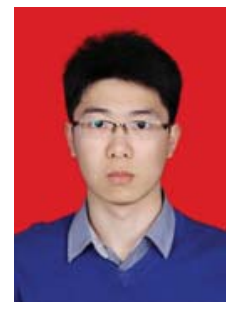

Hongsen Liu is currently working toward the Ph.D. degree in pattern recognition and intelligent systems at the State Key Laboratory of Robotics, Shenyang Institute of Automation, Chinese Academy of Sciences, Shenyang, China. His research interests include pattern recognition, image processing, and robotics.

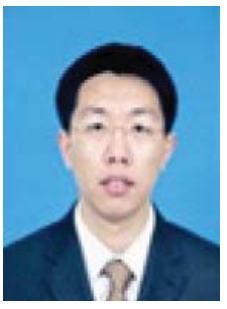

Yang Cong (S'09-M'11-SM'15) is a full professor of Chinese Academy of Sciences. He won the NSFC outstanding youth fund, CAS-Youth Innovation Promotion Association Scholarship. He received the B.Sc. degree from Northeast University in 2004, and the Ph.D. degree from State Key Laboratory of Robotics, Chinese Academy of Sciences in 2009. He was a Research Fellow of National University of Singapore (NUS) and Nanyang Technological University (NTU) from 2009 to 2011, respectively; and a visiting scholar of University of Rochester. He has served on the editorial board of the Journal of Multimedia. His current research interests include image processing, compute vision, machine learning, multimedia, medical imaging, data mining and robot navigation. He has authored over 70 technical papers. He was the PI of more than 15 projects including 4 NSFC projects. He was a senior member of IEEE since 2015.

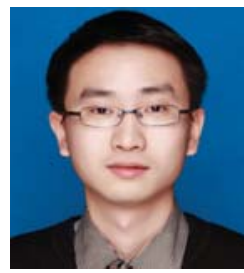

Chenguang Yang (M'10-SM'16) received the Ph.D. degree in control engineering from the National University of Singapore, Singapore, in 2010. From 2009 to 2010, he was a Post-Doctoral Researcher of human robotics with Imperial College London, London, U.K. He is currently a Professor with the Bristol Robotics Laboratory, Bristol, U.K. His research interests include human robot interaction and intelligent system design. Dr. Yang received the EU Marie Curie International Incoming Fellowship, U.K., the EPSRC UKRI Innovation Fellowship, and the Best Paper Award of the IEEE TRANSACTIONS ON ROBOTICS as well as over ten conference Best Paper Awards.

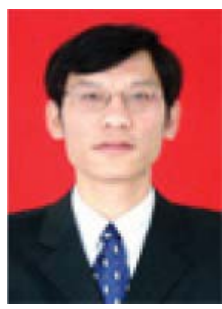

Yandong Tang received the B.S. and M.S.degrees in mathematics from Shandong University, Shandong, China, in 1984 and 1987, respectively, and the Doctors degree in applied mathematics from the University of Bremen, Bremen, Germany, in 2002.He is currently a Professor at the Shenyang Institute of Automation, Chinese Academy of Sciences, Shenyang, China. His research interests include robot vision, image processing, and pattern recognition. 